\title{
Does Delay of Diagnosis and Treatment in Appendicitis Cause Perforation?
}

\author{
Roland E. Andersson ${ }^{1,2}$
}

Published online: 2 March 2016

(C) Société Internationale de Chirurgie 2016

The timing of treatment for appendicitis has been a contentious issue for the past 120 years and still remains unsolved as reported in studies published in various issues of the WJS. The issue has been extensively studied. Most studies have found an association between the pre-hospital delay and the proportion of perforation, and as a consequence also with postoperative morbidity, whereas the impact of in-hospital delay is less clear. A large number of studies have not found any negative impact of in-hospital delay up to 24-36 h. However, studies on the association between delay in the treatment of appendicitis and perforation or morbidity are observed to be with many pitfalls. Many such pitfalls are present in the study by Saar et al [1].

One of the more basic errors is when an association is interpreted as causal. If we see an association between delay and perforation, we think that we can forestall perforation and morbidity if we operate promptly. However, evidence suggest that perforated appendicitis can rarely be prevented. The almost constant findings that perforation is determined by the time from debut of symptoms till the admission to hospital, and that in-hospital delay has no or very limited impact suggest that most perforations occur early before the patient arrives to hospital. The incidence rate (the number per 100,000 inhabitants) of perforations is the same in hospitals applying wide or very strict indication for surgery [2] Differences in management only have an impact on the incidence rate of non-perforated appendicitis. Hospitals that

Roland E. Andersson

roland.andersson@rjl.se

1 Department of Surgery, County Hospital Ryhov, Jönköping, Sweden

2 IKE, Linköping University, Linköping, Sweden operate promptly on all patients with assumed appendicitis will therefore have a low proportion of perforations, as they operate more cases of non-perforated appendicitis, but the number of perforations remains the same. And many studies have shown an association with higher proportion of negative appendectomies in patients operated with short delay.

The total duration of symptoms at operation is mainly determined by the pre-hospital delay. This is associated with structural, organizational and socioeconomic factors like distance to the hospital, health care seeking behaviour, age, sex, race, insurance status, availability of health care, referral system. Most of them can hardly be changed. Older, uninsured and depraved patients tend to seek care late. The association of perforation with race and insurance status has been interpreted as a sign of unequal access to health care, but the evidence are contradicting. Campaigns to motivate the population to seek care early and increase the awareness of the public for symptoms of appendicitis have been tried with no success.

In-hospital delay is associated with organizational factors, like the efficiency of the ER, the triage system, use of guidelines, work load, time of day at admission, availability of radiological service and an emergency operating theatre. Patient-related factors are also involved, like comorbidity, unclear clinical presentation of the disease, time needed for diagnostic workup or resuscitation. The referring practitioner, the ER-physician or the surgeon may also have a part in the delay-the "doctor's delay". This may be because of unclear clinical presentation, miscommunication between the patient and the physician, lack of competence, ignorance or mistake.

It is obvious that a large number of factors may all have an impact on the duration of symptoms at operation and many of them are also interrelated with risk for confounding effects. 
Almost all studies of this issue are cross-sectional, comparing groups of patients with short and long delay. Such studies are however prone to important bias as these groups differ in many characteristics. Patients operated with short delay are more commonly younger, healthier and have more obvious clinical presentation. Patients operated with long delay are more often men, older, have higher grades of comorbidity, depraved, unclear clinical presentation, misdiagnosed, etc. A common mistake is to report on the differences in the proportion of perforation or morbidity between groups with short and long delay, often in small samples, without taking account of the differences in all these characteristics. This is like comparing apples and oranges.

Some studies go further and use more sophisticated analyses, adjusting for the differences in some factors by selection of subgroups of patients with defined characteristics, like "assumed" uncomplicated appendicitis, stratification for characteristics like age, sex, pre-hospital and in-hospital delay or using multivariable logistic regression analysis. Such reports will give less biased estimates, but the mathematical models are imperfect and there will always remain factors that have been overseen and uncontrolled for.

The ideal study design to estimate the effect of delay would be a randomized trial, but no such study has been done. Interventional studies, which are closer to an experimental design and may give less risk of bias, are also rare. One hospital introduced a change in practice not to operate during night time, thus delaying surgery with up to $9 \mathrm{~h}$ for some patients [3]. An analysis comparing the outcome before and after the intervention showed a reduction in the nightly operations but this had no impact on the proportion of perforations, complications or length of stay. Studies from hospitals with a policy not to operate during nighttime compared the outcome in patients operated with short and long delay and found a slightly longer length of stay, but no difference in the proportion of perforations or postoperative complications $[4,5,6]$.

In the end, all these studies have a critical error as they do not take into account the effect of the spontaneous resolution of uncomplicated appendicitis that occur with time. There are strong evidence that support the hypothesis that there are two entities of appendicitis, one progressing to perforation which often occurs within a few hours, and another self-limiting. Self-limiting appendicitis is common and goes often undiagnosed [7]. The rationale for the increasing proportion of perforation with time because of the resolution of undetected, self-limiting appendicitis is nicely illustrated by Luckman (Fig. 1).

Is delay dangerous or is it in fact beneficial? No one would defend or argue for delaying treatment in patients with advanced appendicitis. Early identification and treatment of perforated appendicitis is therefore important. But

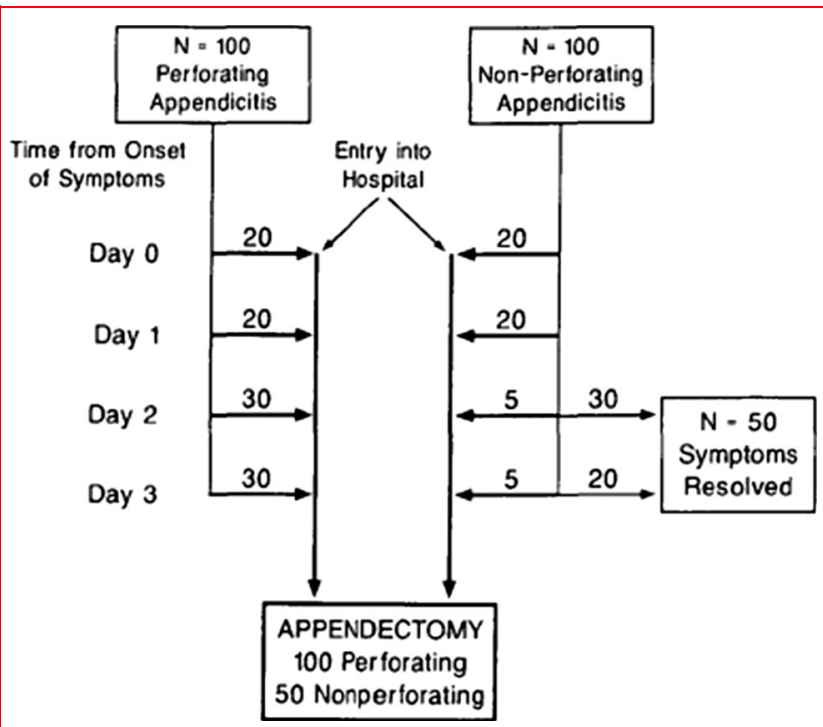

Fig. 1 Time course from the onset of symptoms to hospital admission for two hypothetical types of appendicitis. Numbers over arrows indicate cases admitted to the hospital on the day after symptom onset shown on the same line (from Luckman [8])

some hours delay in patients with simple appendicitis is safe and may in fact be beneficial as it may allow spontaneous resolution to occur. As a consequence the proportion of perforations will increase due to the selection as the advanced cases will remain. In patients with an equivocal diagnosis, active observation is a time-proven, safe and simple management which gives an improved diagnostic accuracy.

\section{References}

1. Talving P, Saar S, Laos J et al (2016) Delay between onset of symptoms and surgery in acute appendicitis increases perioperative morbidity: a prospective study. World J Surg. doi:10.1007/ s00268-016-3416-2

2. Andersson R, Hugander A, Thulin A et al (1994) Indications for operation in suspected appendicitis and incidence of perforation. BMJ 308:107-110

3. Schnüriger B, Laue J, Kröll D et al (2014) Introduction of a new policy of no nighttime appendectomies: impact on appendiceal perforation rates and postoperative morbidity. World J Surg 38:18-24. doi:10.1007/s00268-013-2225-0

4. Surana R, Quinn F, Puri P (1993) Is it necessary to perform appendicectomy in the middle of the night in children? BMJ 306:1168

5. Yardeni D, Hirschl RB, Drongowski RA et al (2004) Delayed versus immediate surgery in acute appendicitis: do we need to operate during the night? J Pediatr Surg 39:464-469

6. Taylor M, Emil S, Nguyen N, Ndiforchu F (2005) Emergent vs urgent appendectomy in children: a study of outcomes. J Pediatr Surg 40:1912-1915 
7. Andersson RE (2007) The natural history and traditional management of appendicitis revisited: spontaneous resolution and predominance of prehospital perforations imply that a correct diagnosis is more important than an early diagnosis. World $\mathrm{J}$ Surg 31:86-92. doi:10.1007/s00268-016-3416-2
8. Luckmann R (1989) Incidence and case fatality rates for acute appendicitis in California: a population-based study of the effects of age. Am J Epidemiol 129:905-918 